\title{
CONTROLE DE Euphorbia heterophylla E Ipomoea grandifolia COM A UTILIZAÇÃo DE GlYPHOSATE ISOlAdo OU EM ASSOCIAÇÃo COM LATIFOLICIDAS ${ }^{1}$
}

\author{
Control of Euphorbia heterophylla and Ipomoea grandifolia Using Glyphosate Isolated or in \\ Association with Broadleaf Herbicides
}

\author{
RAMIRES, A.C. ${ }^{2}$, CONSTANTIN, J. ${ }^{3}$, OLIVEIRA JR., R.S. ${ }^{4}$, GUERRA, N. ${ }^{4}$, ALONSO, D.G. ${ }^{5}$ e \\ BIFFE, D.F. ${ }^{5}$
}

\begin{abstract}
RESUMO - No controle de plantas daninhas em pós-emergência na cultura da soja RR $^{\circledR}$, misturas de latifolicidas com o glyphosate têm sido utilizadas principalmente para melhorar a eficácia sobre espécies de dificil controle, a fim de se obter controle residual ou prevenir o surgimento de resistência. Este trabalho teve o objetivo de avaliar a eficácia de glyphosate isolado ou em mistura com latifolicidas para o controle de Euphorbia heterophylla e Ipomoea grandifolia, em dois estádios de desenvolvimento (1 a 3 folhas e 4 a 6 folhas). Para isso, foram realizados quatro experimentos em casa de vegetação, com glyphosate nas doses de 480 e 960 ge.a. ha-1, isolado ou combinado com os latifolicidas cloransulam-methyl $\left(30,24 \mathrm{~g} \mathrm{ha}^{-1}\right)$, chlorimuron-ethyl $\left(12,5 \mathrm{~g} \mathrm{ha}^{-1}\right)$, imazethapyr (80 $\left.\mathrm{g} \mathrm{ha}^{-1}\right)$, fomesafen $\left(62,5 \mathrm{~g} \mathrm{ha}^{-1}\right)$, lactofen $\left(72,0 \mathrm{~g} \mathrm{ha}^{-1}\right)$, flumiclorac-pentyl (30,0 $\left.\mathrm{g} \mathrm{ha}^{-1}\right)$ e bentazon (480 $\left.\mathrm{g} \mathrm{ha}^{-1}\right)$, em delineamento inteiramente casualizado, com quatro repetições. Em ambas as espécies, a utilização da dose de 960 ge.a. ha ${ }^{-1}$ do glyphosate dispensa a necessidade de misturas com outros herbicidas quando os tratamentos são aplicados no estádio de 1-3 folhas. Para aplicações realizadas em $E$. heterophylla no estádio de 4-6 folhas, aumentos da eficácia de controle em relação ao glyphosate aplicado isoladamente a $480 \mathrm{~g}$ e.a. ha-1 foram observados com as misturas com inibidores de protox. Para I. grandifolia em estádio de 4-6 folhas, os herbicidas fomesafen e flumiclorac-pentyl reduziram a eficácia de controle quando adicionados ao glyphosate a 480 g e.a. ha ${ }^{-1}$. Já as misturas contendo glyphosate a 960 g e.a. ha ${ }^{-1}$ proporcionaram incrementos da eficácia de controle. De forma geral, o controle das espécies E. heterophylla e I. grandifolia foi melhor quando os tratamentos foram aplicados no estádio de 1-3 folhas tanto pelo glyphosate (480 e 960 e.a. $\mathrm{g} \mathrm{ha}^{-1}$ ) isolado como em mistura com os demais herbicidas.
\end{abstract}

Palavras-chave: corda-de-viola, Glycine max, leiteiro, herbicidas, soja tolerante ao glyphosate.

\begin{abstract}
Mixtures of broadleaf herbicides and glyphosate have been adopted during postemergence weed control in $R R \otimes$ soybeans mainly to improve the efficacy of this herbicide when applied on tolerant weeds, to provide residual weed control or to prevent the selection of resistant biotypes. This work aimed to evaluate the efficacy of glyphosate alone or in tank mixtures with other broadleaf herbicides to control Euphorbia heterophylla and Ipomoea grandifolia, at two growth stages (1-to-3 leaves and 4-to-6 leaves). Thus, four greenhouse assays were carried out: glyphosate (480 and $960 \mathrm{~g}$ a.e. $\mathrm{ha}^{-1}$ ) applied alone or combined in tank mixtures with chloransulammethyl (30.24 $\left.\mathrm{g} \mathrm{ha}^{-1}\right)$, chlorimuron-ethyl (12.5 $\left.\mathrm{g} \mathrm{ha}^{-1}\right)$, imazethapyr (80 $\mathrm{g} \mathrm{ha}$ ), fomesafen (62.5 $\left.\mathrm{g} \mathrm{ha} \mathrm{a}^{-1}\right)$, lactofen (72.0 $\left.\mathrm{g} \mathrm{ha}^{-1}\right)$, flumiclorac-pentyl (30.0 $\left.\mathrm{g} \mathrm{ha}^{-1}\right)$ and bentazon (480 $\left.\mathrm{g} \mathrm{ha}^{-1}\right)$. All experiments were
\end{abstract}

Recebido para publicação em 24.9.2009 e na forma revisada em 3.9.2010.

2 Engo-Agro ${ }^{\circ}$, M.Sc., representante técnico e comercial da Cooperativa Agroindustrial no Município de Floresta - COCAMAR, Estado do Paraná, <antonio.ramires@cocamar.com.br>; ${ }^{3}$ Professor Associado, Núcleo de Estudos Avançados em Ciência das Plantas Daninhas - NAPD/UEM, Dep. de Agronomia, Universidade Estadual de Maringá, Av. Colombo, 5790, 87020-9000, Maringá-PR; ${ }^{4}$ Eng-a-Agr ${ }^{\text {. }}$, Mestranda do Curso de Pós-Graduação em Agronomia na área de Proteção de Plantas, NAPD/UEM; ${ }^{5}$ Eng ${ }^{0}$-Agr ${ }^{\circ}$., MSc., Doutorando do Curso de Pós-Graduação em Agronomia na área de Proteção de Plantas, NAPD/UEM. 
arranged in a randomized block design, with four replicates. For both weeds, the use of $960 \mathrm{~g}$ ha-1 of glyphosate alone was sufficient, when application was performed at 1-3 leaf stage. For applications performed at 4-6 leaf stage for $\boldsymbol{E}$. heterophylla, increased weed control in relation to glyphosate applied alone can be achieved by using mixtures with protox inhibitors. In relation to I. grandifolia at the 46 leaf stage, fomesafen and flumiclorac-penthyl decreased weed control when added to glyphosate at $480 \mathrm{~g}$ a.e. $h^{-1}$. On the other hand, mixtures containing glyphosate at $960 \mathrm{~g}$ a.e. ha ${ }^{-1}$ provided increased weed control. In general, control of $\boldsymbol{E}$. heterophylla and I. grandifolia was improved when herbicide treatments were applied at 1-3 leaf stage, either using glyphosate alone or mixed with other broadleaf herbicides.

Keywords: morningglory, Glycine max, wild poinsettia, herbicides, glyphosate resistant soybean.

\section{INTRODUÇÃO}

A introdução no mercado brasileiro das variedades de soja transgênicas que possuem o gene de resistência ao herbicida glyphosate gerou importantes modificações no controle químico de plantas daninhas (Gazziero et al., 2006). Devido às características positivas do herbicida glyphosate, como ação em pós-emergência tanto inicial como tardia, efeito sistêmico e amplo espectro de ação, muitos agricultores passaram a acreditar que os problemas no manejo de plantas daninhas na cultura da soja estariam resolvidos com a introdução das variedades Roundup Ready ${ }^{\circledR}\left(R R^{\circledR}\right)$. No entanto, o controle insatisfatório de algumas espécies de plantas daninhas em aplicações de dessecação, no sistema de plantio direto com esse herbicida, já mostrava indícios de que, mesmo sendo um excelente produto, associações com outros princípios ativos poderiam ser necessárias (Vidal et al., 2003; Petter et al., 2007). Resultados de pesquisa têm demonstrado que o uso combinado de glyphosate com herbicidas aplicados no manejo de plantas daninhas em soja convencional aumenta o espectro e a eficácia de controle de plantas consideradas mais tolerantes à ação do glyphosate isolado (Gonzini et al., 1999; Kranz et al., 2001; Monquero et al., 2001; Vidrine et al., 2002; Norsworthy \& Grey, 2004; Procópio et al., 2007).

Para controle de plantas daninhas em pósemergência na soja $R^{\circledR}$, misturas de latifolicidas com o glyphosate têm sido utilizadas principalmente para melhorar a eficácia sobre espécies de difícil controle, a fim de obter controle residual ou prevenir o surgimento de resistência. No entanto, misturas em tanque podem apresentar resultados diferentes dos obtidos com o uso dos produtos isoladamente, tanto para a eficiência de controle da espécie daninha quanto para a seletividade à cultura. Misturas de glyphosate com latifolicidas aplicados em pós-emergência do cultivar de soja CD $214 \mathrm{RR}$ afetaram significativamente a seletividade para a cultura (Alonso, 2008).

Shaw \& Arnold (2002) estudaram a eficiência de glyphosate em aplicações isoladas e em mistura com acifluorfen, chlorimuron, cloransulam-methyl, fomesafen, imazaquin e pyrithiobac no controle de Brachiaria platyphylla, Sesbania exaltata, Sorghum halepense e Ipomoea lacunosa. Esses autores observaram que, ao adicionar acifluorfen ao glyphosate, o controle de $S$. exaltata atingiu $100 \%$ contra $55 \%$ com o uso do glyphosate isoladamente. Da mesma forma, a adição de fomesafen ou acifluorfen ao glyphosate elevou o controle de I. lacunosa de $67 \%$ para $90 \%$ e $98 \%$, respectivamente. Já o controle de B. platyphylla e $S$. halepense não apresentou incremento para as misturas utilizadas.

Outro fator de grande relevância para obtenção de resultados positivos no controle de plantas infestantes é o estádio de desenvolvimento. Gazziero et al. (2006), ao estudarem a deposição de glyphosate em plantas de $E$. heterophylla, concluíram que o depósito da calda de pulverização reduziu progressivamente com o desenvolvimento das plantas, sugerindo que para garantir maior eficiência de controle as aplicações devem ser realizadas em estádios mais precoces.

Procópio et al. (2007) constataram baixa eficiência no controle das plantas daninhas 
E. heterophylla (4-8 folhas), Commelina benghalensis (2-6 folhas) e I. grandifolia (2-6 folhas) com a aplicação isolada de glyphosate nas doses de 480, 960 e 1.440 g e.a. ha ${ }^{-1}$, porém 1.440 g e.a. ha-1 de glyphosate associado a imazethapyr (50,0 ou 100,0 $\mathrm{g} \mathrm{ha}^{-1}$ ) proporcionaram melhor controle para E. heterophylla, ao passo que a associação com chlorimuronethyl (5,0 ou 10,0 $\left.\mathrm{g} \mathrm{ha}^{-1}\right)$ foi mais eficiente para C. benghalensis e I. grandifolia.

Com base no exposto e na necessidade de estudos sobre misturas com o uso de glyphosate, objetivou-se neste trabalho verificar a eficácia do glyphosate isolado e em misturas com latifolicidas no controle de Euphorbia heterophylla e Ipomoea grandifolia em dois estádios de desenvolvimento.

\section{MATERIAL E MÉTODOS}

Os experimentos foram conduzidos nos anos de 2007 e 2008, em casa de vegetação localizada no município de Maringá-PR, em latitude de $23^{\circ} 24^{\prime} 28^{\prime \prime}$ S e longitude de $51^{\circ} 56^{\prime} 48^{\prime \prime}$ $\mathrm{W}$, com altitude de $572 \mathrm{~m}$.

O solo utilizado como substrato foi classificado como franco-argiloarenoso, sendo constituído por $71,0 \%$ de areia, $21,0 \%$ de argila e $8,0 \%$ de silte. Com relação às características químicas, apresentou $\mathrm{pH}$ de 4,5 em $\mathrm{CaCl}_{2} ; 4,96 \mathrm{cmol}_{\mathrm{c}} \mathrm{dm}^{-3}$ de $\mathrm{H}^{+}+\mathrm{Al}^{3+}$; $0,97 \mathbf{c m o l}_{\mathrm{c}} \mathrm{dm}^{-3}$ de $\mathrm{Ca}^{+2} ; 0,78 \mathrm{cmol}_{\mathrm{c}} \mathrm{dm}^{-3} \mathrm{de}$ $\mathrm{Mg}^{+2} ; 0,24 \mathrm{cmol}_{\mathrm{c}} \mathrm{dm}^{-3}$ de K $\mathrm{K}^{+} ; 19,2 \mathrm{mg} \mathrm{dm}^{-3}$ de $\mathrm{P}$; e $13,68 \mathrm{~g} \mathrm{dm}^{-3} \mathrm{de} \mathrm{C}_{\text {. }}$

As unidades experimentais foram constituídas por vasos de polietileno com capacidade de $3 \mathrm{dm}^{3}$ de solo, nos quais foram semeadas 50 sementes de Euphorbia heterophylla ou Ipomoea grandifolia à profundidade de $1,5 \mathrm{~cm}$, sendo elas submetidas à irrigação diária. Após emergência e estabelecimento completo, foi realizado o desbaste delas, a fim de se obter um número de 10 plantas de cada espécie por unidade experimental.

A semeadura de E. heterophylla e I. grandifolia foi feita em 1/12/2007, e as aplicações dos tratamentos foram efetuadas em dois estádios de desenvolvimento das plantas daninhas, sendo o primeiro com plantas apresentando desenvolvimento vegetativo entre 1 e 3 folhas e o segundo com plantas entre
4 e 6 folhas, nos dias 15/12/2007 e 4/1/2008, respectivamente.

O equipamento utilizado na aplicação dos produtos foi um pulverizador costal pressurizado à base de $\mathrm{CO}_{2}$, equipado com barra contendo três pontas tipo leque XR 110-02, com pressão constante de $2,0 \mathrm{kgf} \mathrm{cm}^{-2}$ e volume de calda equivalente a $200 \mathrm{~L} \mathrm{ha}^{-1}$. As condições climáticas para ambas as aplicações foram semelhantes, com temperatura entre 21,0 e $24,5^{\circ} \mathrm{C}$, umidade relativa do ar média de $82 \%$, céu claro, solo úmido e vento com velocidade de aproximadamente $2 \mathrm{~km} \mathrm{~h}^{-1}$.

O delineamento experimental foi o inteiramente casualizado, com quatro repetições. Foram conduzidos simultaneamente quatro experimentos isolados, para cada espécie e estádio de aplicação dos herbicidas. Os herbicidas testados foram glyphosate nas doses de 480 e 960 g e.a. ha ${ }^{-1}$, em aplicações isoladas ou combinadas com cloransulam-methyl $\left(30,24 \mathrm{~g} \mathrm{ha}^{-1}\right)$, chlorimuron-ethyl $\left(12,5 \mathrm{~g} \mathrm{ha}^{-1}\right)$, imazethapyr $\left(80 \mathrm{gha}^{-1}\right)$, fomesafen $\left(62,5 \mathrm{~g} \mathrm{ha}^{-1}\right)$, lactofen $\left(72,0 \mathrm{~g} \mathrm{ha}^{-1}\right)$, flumiclorac-pentyl (30,0 $\mathrm{g} \mathrm{ha}^{-1}$ ) e bentazon (480 $\mathrm{g} \mathrm{ha}^{-1}$ ), além de uma testemunha sem aplicação, totalizando 17 tratamentos.

As avaliações de controle foram realizadas aos 3, 7, 14, 21, 28 e 35 dias após aplicação (DAA) para todos os tratamentos. Os critérios utilizados nas avaliações foram $0 \%$ de controle para as plantas sem aplicação dos produtos (testemunha sem aplicação) e 100\% de controle para a morte de todas as plantas; as notas foram obtidas por meio de avaliação visual, conforme procedimentos da SBCPD (1995). Aos 35 DAA, as plantas foram cortadas rente ao solo, embaladas em sacos de papel e secadas em estufa de circulação forçada de ar a $70^{\circ} \mathrm{C}$ por 12 horas, quando foi determinada a massa seca da parte aérea, por pesagem em balança de precisão.

Os dados obtidos das avaliações foram submetidos à análise de variância pelo teste $\mathrm{F}$, e as médias, comparadas pelo teste de Scott \& Knott (1974) a 5\% de probabilidade. Quanto à massa seca da parte aérea, os dados foram transformados em $\sqrt{X+0,5}$, e a comparação foi feita utilizando os valores originais (g por vaso). 


\section{RESULTADOS E DISCUSSÃO}

\section{Euphorbia heterophylla no estádio de 1 a 3 folhas}

Os valores de porcentagem de controle e massa seca da parte aérea para as plantas de $E$. heterophylla encontram-se na Tabela 1. Observa-se que o glyphosate, quando aplicado isoladamente nas doses de 480 e 960 g e.a. ha ${ }^{-1}$, mostrou excelente nivel de controle (acima de $90 \%$ ) a partir de 21 e 14 DAA, respectivamente, o que demonstra maior velocidade inicial de controle para a maior dose.

Aos 35 DAA, as misturas de glyphosate (480 g e.a. ha ${ }^{-1}$ ) com fomesafen, flumicloracpentyl, bentazon, lactofen e imazethapyr apresentaram menor controle de E. heterophylla, em relação ao glyphosate isoladamente, demonstrando interação negativa. Da mesma forma, Monquero et al. (2001) observaram efeito negativo da mistura de glyphosate com bentazon e sulfentrazone para o controle de Galinsoga parviflora e Richardia brasiliensis, respectivamente. Para as misturas com cloransulam-methyl e chlorimuron-ethyl, não houve diferenças significativas em relação à menor dose de glyphosate isolado. Vidal et al. (2003) verificaram efeitos negativos ao testarem a associação de glyphosate nas doses de 540, 720 e 900 g e.a. ha ${ }^{-1}$ com simazine + atrazine $\left(1.750+1.750 \mathrm{~g} \mathrm{ha}^{-1}\right)$ em plantas de sorgo, as quais apresentaram maior teor de clorofila para os tratamentos onde houve a associação dos produtos, quando comparado ao glyphosate isolado.

O uso de glyphosate isolado a 960 g e.a. ha-1 ou associado aos demais herbicidas avaliados demonstrou controle muito eficiente de E. heterophylla, não havendo diferenças significativas entre esses tratamentos.

Todos os tratamentos herbicidas proporcionaram redução significativa da massa seca da parte aérea de E. heterophylla, porém os herbicidas fomesafen e bentazon associados com o glyphosate a 480 g e.a. ha ${ }^{-1}$ proporcionaram redução menos intensa que os demais tratamentos (Tabela 1).

Tabela 1 - Porcentagens de controle aos 3, 7, 14, 21, 28 e 35 DAA (dias após aplicação) e massa seca da parte aérea de Euphorbia heterophylla obtidas após aplicação de diferentes tratamentos contendo glyphosate no estádio de 1 a 3 folhas - Maringá-PR, $2007 / 2008$

\begin{tabular}{|c|c|c|c|c|c|c|c|c|c|}
\hline Tratamento & Dose & \multicolumn{6}{|c|}{$\%$ Controle } & \multicolumn{2}{|c|}{ Massa seca da parte aérea } \\
\hline Ingrediente ativo & (g i.a. ou e.a. ha $\left.{ }^{-1}\right)$ & $3 \mathrm{DAA}$ & $7 \mathrm{DAA}$ & 14 DAA & $21 \mathrm{DAA}$ & 28 DAA & 35 DAA & g por vaso & $\begin{array}{c}\% \text { em } \\
\text { relação à } \\
\text { testemunha }\end{array}$ \\
\hline Testemunha sem aplicação & - & $0,00 \mathrm{~g}$ & $0,00 \mathrm{f}$ & $0,00 \mathrm{f}$ & $0,00 \mathrm{~g}$ & $0,00 \mathrm{f}$ & $0,00 \mathrm{e}$ & $1,47 \mathrm{a}(2,12)$ & 100 \\
\hline Glyphosate & 480 & $6,25 \mathrm{f}$ & $25,00 \mathrm{~d}$ & $76,75 \mathrm{~b}$ & $93,25 \mathrm{a}$ & $98,00 \mathrm{~b}$ & $98,25 \mathrm{a}$ & $0,73 \mathrm{c}(0,03)$ & 1 \\
\hline Cloransulam-methyl + Glyphosate & $30,24+480$ & $13,75 \mathrm{e}$ & $34,75 \mathrm{c}$ & $72,50 \mathrm{c}$ & $95,75 \mathrm{a}$ & $99,50 \mathrm{a}$ & $99,50 \mathrm{a}$ & $0,71 \mathrm{c}(0,01)$ & 0 \\
\hline Chlorimuron-ethyl + Glyphosate & $12,50+480$ & $12,50 \mathrm{e}$ & $31,25 \mathrm{c}$ & $73,25 \mathrm{c}$ & $85,00 \mathrm{~b}$ & $96,00 \mathrm{a}$ & $97,50 \mathrm{a}$ & $0,74 \mathrm{c}(0,05)$ & 2 \\
\hline Imazethapyr + Glyphosate & $80+480$ & $6,25 \mathrm{f}$ & $5,50 \mathrm{e}$ & $48,75 \mathrm{~d}$ & $68,75 \mathrm{~d}$ & $87,75 \mathrm{c}$ & $92,25 \mathrm{~b}$ & $0,82 \mathrm{c}(0,17)$ & 8 \\
\hline Fomesafen + Glyphosate & $62,50+480$ & $25,00 \mathrm{~d}$ & $60,50 \mathrm{~b}$ & $63,75 \mathrm{c}$ & $60,00 \mathrm{e}$ & $72,00 \mathrm{e}$ & $73,25 \mathrm{~d}$ & $1,20 \mathrm{~b}(0,95)$ & 45 \\
\hline Lactofen + Glyphosate & $72+480$ & $40,00 \mathrm{~b}$ & $73,25 \mathrm{a}$ & $68,75 \mathrm{c}$ & $76,25 \mathrm{c}$ & $82,00 \mathrm{~d}$ & $83,00 \mathrm{c}$ & $0,96 \mathrm{c}(0,44)$ & 21 \\
\hline Flumiclorac-pentyl + Glyphosate & $30+480$ & $33,75 \mathrm{c}$ & $60,50 \mathrm{~b}$ & $67,50 \mathrm{c}$ & $69,50 \mathrm{~d}$ & $79,50 \mathrm{~d}$ & $75,75 \mathrm{~d}$ & $0,92 \mathrm{c}(0,37)$ & 17 \\
\hline Bentazon + Glyphosate & $480+480$ & $7,50 \mathrm{f}$ & $7,50 \mathrm{e}$ & $15,00 \mathrm{e}$ & $41,75 \mathrm{f}$ & $71,25 \mathrm{e}$ & $75,25 \mathrm{~d}$ & $1,13 \mathrm{~b}(0,80)$ & 38 \\
\hline Glyphosate & 960 & $12,50 \mathrm{e}$ & $38,75 \mathrm{c}$ & $95,00 \mathrm{a}$ & $99,50 \mathrm{a}$ & $99,50 \mathrm{a}$ & $99,75 \mathrm{a}$ & $0,71 \mathrm{c}(0,00)$ & 0 \\
\hline Cloransulam-methyl + Glyphosate & $30,24+960$ & $12,50 \mathrm{e}$ & $23,75 \mathrm{~d}$ & $82,50 \mathrm{~b}$ & $99,50 \mathrm{a}$ & $100,00 \mathrm{a}$ & $100,00 \mathrm{a}$ & $0,71 \mathrm{c}(0,00)$ & 0 \\
\hline Chlorimuron-ethyl + Glyphosate & $12,50+960$ & $8,75 \mathrm{f}$ & $35,75 \mathrm{~d}$ & $85,75 \mathrm{~b}$ & $99,50 \mathrm{a}$ & $100,00 \mathrm{a}$ & $100,00 \mathrm{a}$ & $0,71 \mathrm{c}(0,00)$ & 0 \\
\hline Imazethapyr + Glyphosate & $80+960$ & $8,75 \mathrm{f}$ & $35,00 \mathrm{c}$ & $85,00 \mathrm{~b}$ & $98,75 \mathrm{a}$ & $100,00 \mathrm{a}$ & $100,00 \mathrm{a}$ & $0,71 \mathrm{c}(0,00)$ & 0 \\
\hline Fomesafen + Glyphosate & $62,50+960$ & $46,25 \mathrm{a}$ & $68,25 \mathrm{a}$ & $79,50 \mathrm{~b}$ & $95,25 \mathrm{a}$ & $96,50 \mathrm{a}$ & $97,50 \mathrm{a}$ & $0,74 \mathrm{c}(0,05)$ & 2 \\
\hline Lactofen + Glyphosate & $72+960$ & $45,00 \mathrm{a}$ & $69,50 \mathrm{a}$ & $81,25 \mathrm{~b}$ & $96,25 \mathrm{a}$ & $98,00 \mathrm{a}$ & $98,50 \mathrm{a}$ & $0,72 \mathrm{c}(0,02)$ & 1 \\
\hline Flumiclorac-pentyl + Glyphosate & $30+960$ & $33,75 \mathrm{c}$ & $69,50 \mathrm{a}$ & $83,75 \mathrm{~b}$ & $93,75 \mathrm{a}$ & $96,75 \mathrm{a}$ & $97,50 \mathrm{a}$ & $0,72 \mathrm{c}(0,02)$ & 1 \\
\hline Bentazon + Glyphosate & $480+960$ & $13,75 \mathrm{e}$ & $37,25 \mathrm{~d}$ & $80,75 \mathrm{~b}$ & $99,50 \mathrm{a}$ & $99,75 \mathrm{a}$ & $99,75 \mathrm{a}$ & $0,71 \mathrm{c}(0,01)$ & 0 \\
\hline $\mathrm{CV}(\%)$ & & 16,62 & 9,76 & 6,92 & 5,98 & 2,42 & 3,33 & 23,12 & \\
\hline
\end{tabular}

Valores seguidos de mesma letra nas colunas não diferem entre si pelo teste de Scott Knott $\mathrm{p}<0,05$.

Os valores da massa seca analisados estão transformados em $\sqrt{X+0,5}$ e os valores entre parênteses são as médias originais. 
Herbicidas com rápida ação de contato como os inibidores de protox avaliados neste trabalho - podem afetar a absorção foliar e a translocação de herbicidas sistêmicos, como o glyphosate. O fato de o controle de $E$. heterophylla ter sido semelhante ou, mais comumente, superior nas misturas com glyphosate na dose de $960 \mathrm{~g}$ e.a. ha ${ }^{-1}$ reforça a possibilidade de que os efeitos negativos das misturas contendo glyphosate a 480 g e.a. ha ${ }^{-1}$ estejam associados à interação negativa entre os herbicidas.

Nota-se por esses resultados que, para o controle de $E$. heterophylla, no estádio de 1 a 3 folhas, o glyphosate aplicado isoladamente nas duas doses testadas apresentou elevada eficiência; a utilização de misturas apresentou desempenho semelhante ou inferior ao do glyphosate isoladamente.

\section{Euphorbia heterophylla no estádio de 4 a 6 folhas}

Na Tabela 2 encontram-se as porcentagens de controle e massa seca da parte aérea das plantas de $E$. heterophylla no estádio de 4 a 6 folhas. Nota-se que os resultados de controle tiveram acréscimos com o aumento da dose de glyphosate.

Em relação ao controle final (35 DAA) de E. heterophylla, tratamentos com glyphosate (480 g e.a. ha ${ }^{-1}$ ) em mistura com cloransulammethyl, chlorimuron-ethyl e imazethapyr não diferiram significativamente do tratamento com glyphosate isolado; já as misturas com fomesafen, lactofen e flumiclorac-penthyl evidenciaram acréscimos de controle quando comparadas à aplicação somente do glyphosate. $\mathrm{O}$ herbicida bentazon em mistura com glyphosate teve um controle $20 \%$ menor que o do glyphosate isolado, demonstrando uma interação negativa entre esses produtos. Os resultados de controle de $E$. heterophylla obtidos com glyphosate isolado no presente trabalho são superiores aos encontrados por Procópio et al. (2007), que constataram baixa eficiência $(<55 \%)$ no controle de $E$. heterophylla (4-8 folhas) na aplicação de doses de 480, 960 e 1.440 g e.a. ha ${ }^{-1}$. No entanto, quando o glyphosate ( 1.440 g e.a. ha $\left.{ }^{-1}\right)$ foi associado a imazethapyr $\left(50,0\right.$ ou $\left.100,0 \mathrm{~g} \mathrm{ha}^{-1}\right)$ o controle desta espécie foi de $100 \%$ - resultado semelhante ao obtido neste trabalho.
Aplicações de glyphosate a 960 g e.a. ha ${ }^{-1}$ em mistura com os demais produtos testados mostraram maior eficiência no controle de $E$. heterophylla no estádio de 4 a 6 folhas, exceto para as misturas com cloransulam-methyl e flumiclorac-pentyl.

No que se refere à massa seca da parte aérea, os tratamentos apresentaram valores entre 14 e $35 \%$ e entre 1 e $11 \%$ em relação à testemunha sem aplicação, respectivamente para as misturas de glyphosate com os demais herbicidas nas doses de 480 e 960 g e.a. ha ${ }^{-1}$ (Tabela 2), mostrando novamente que, de modo geral, a aplicação de maiores doses de glyphosate contribui para melhor eficiência de controle das plantas de E. heterophylla em estádios mais avançados.

As plantas de E. heterophylla foram mais bem controladas quando os herbicidas foram aplicados sobre as plantas mais jovens com 1-3 folhas (Tabelas 1 e 2), corroborando estudo de Gazziero et al. (2006), os quais concluíram que o depósito da calda de pulverização em plantas de $E$. heterophylla é reduzido progressivamente com o desenvolvimento das plantas, sugerindo maior eficiência de controle quando as aplicações são feitas em estádios mais precoces. Ferreira et al. (2003) também observaram que nestas plantas existem barreiras foliares que impedem a penetração do herbicida, devido ao alto teor de cera epicuticular, à alta densidade de laticíferos e à grande espessura da cutícula, que provavelmente é mais pronunciada quando as plantas se encontram em estádio de desenvolvimento mais avançado.

\section{Ipomoea grandifolia no estádio de 1 a 3 folhas}

As porcentagens de controle e a massa seca da parte aérea relativas às plantas de I. grandifolia estão dispostas na Tabela 3.

Os controles proporcionados pela menor dose de glyphosate (480 g e.a. ha-1) em mistura com cloransulam-methyl, lactofen e chlorimuron-ethyl foram superiores ao glyphosate aplicado isoladamente, aos 35 DAA, com $88,75,94,75$ e $87,25 \%$, respectivamente. Por outro lado, nas misturas dessa mesma dose de glyphosate com fomesafen, flumicloracpentyl e bentazon percebe-se queda acentuada 
Tabela 2 - Porcentagens de controle aos 3, 7, 14, 21, 28 e 35 DAA (dias após aplicação) e massa seca da parte aérea de Euphorbia heterophylla obtidas após aplicação de diferentes tratamentos contendo glyphosate no estádio de 4 a 6 folhas Maringá-PR, 2007/2008

\begin{tabular}{|c|c|c|c|c|c|c|c|c|c|}
\hline Tratamento & Dose & \multicolumn{6}{|c|}{$\%$ Controle } & \multicolumn{2}{|c|}{ Massa seca da parte aérea } \\
\hline Ingrediente ativo & $\left(\right.$ g i.a. ou e.a. ha $\left.{ }^{-1}\right)$ & 3 DAA & $7 \mathrm{DAA}$ & 14 DAA & $21 \mathrm{DAA}$ & 28 DAA & 35 DAA & $\mathrm{g}$ por vaso & $\begin{array}{c}\% \text { em } \\
\text { relação à } \\
\text { testemunha }\end{array}$ \\
\hline Testemunha sem aplicação & - & $0,00 \mathrm{e}$ & $0,00 \mathrm{~g}$ & $0,00 \mathrm{f}$ & $0,00 \mathrm{f}$ & $0,00 \mathrm{f}$ & $0,00 \mathrm{f}$ & $2,38 \mathrm{a}(5,25)$ & 100 \\
\hline Glyphosate & 480 & $36,25 \mathrm{~b}$ & $48,75 \mathrm{e}$ & $79,50 \mathrm{~d}$ & $84,00 \mathrm{c}$ & $67,50 \mathrm{c}$ & $67,50 \mathrm{~d}$ & 1,22 c $(0,99)$ & 19 \\
\hline Cloransulam-methyl + Glyphosate & $30,24+480$ & $22,50 \mathrm{c}$ & $48,75 \mathrm{e}$ & $79,00 \mathrm{~d}$ & $80,00 \mathrm{~d}$ & $65,00 \mathrm{~d}$ & $60,00 \mathrm{~d}$ & $1,24 \mathrm{c}(1,05)$ & 20 \\
\hline Chlorimuron-ethyl + Glyphosate & $12,50+480$ & $45,00 \mathrm{a}$ & $82,00 \mathrm{~b}$ & $87,00 \mathrm{c}$ & $90,75 \mathrm{~b}$ & $81,25 \mathrm{~b}$ & $80,50 \mathrm{c}$ & $1,10 \mathrm{c}(0,73)$ & 14 \\
\hline Imazethapyr + Glyphosate & $80+480$ & $52,50 \mathrm{a}$ & $85,50 \mathrm{a}$ & $88,25 \mathrm{c}$ & $96,25 \mathrm{a}$ & $82,00 \mathrm{~b}$ & $84,75 \mathrm{~b}$ & $1,09 \mathrm{c}(0,71)$ & 14 \\
\hline Fomesafen + Glypho sate & $62,50+480$ & $12,50 \mathrm{~d}$ & $47,50 \mathrm{e}$ & $75,00 \mathrm{e}$ & $72,50 \mathrm{e}$ & $57,50 \mathrm{e}$ & $63,75 \mathrm{~d}$ & $1,33 \mathrm{~b}(1,27)$ & 24 \\
\hline Lactofen + Glyphosate & $72+480$ & $45,00 \mathrm{a}$ & $85,00 \mathrm{a}$ & $88,25 \mathrm{c}$ & $90,75 \mathrm{~b}$ & $73,75 \mathrm{c}$ & $77,75 \mathrm{c}$ & $1,20 \mathrm{c}(0,95)$ & 18 \\
\hline Flumiclorac-pentyl + Glypl & $30+480$ & $7,50 \mathrm{~d}$ & $33,75 \mathrm{f}$ & $73,00 \mathrm{e}$ & $74,50 \mathrm{e}$ & $45,00 \mathrm{e}$ & $47,50 \mathrm{e}$ & $1,51 \mathrm{~b}(1,85)$ & 35 \\
\hline Bentazon + Glyphosate & $480+480$ & $15,00 \mathrm{~d}$ & $55,00 \mathrm{~d}$ & $77,00 \mathrm{e}$ & $78,75 \mathrm{~d}$ & $63,75 \mathrm{~d}$ & $66,25 \mathrm{~d}$ & $1,31 \mathrm{~b}(1,23)$ & 23 \\
\hline Glyphosate & 960 & $38,75 \mathrm{~b}$ & $76,25 \mathrm{~b}$ & $92,50 \mathrm{~b}$ & $94,25 \mathrm{a}$ & $92,50 \mathrm{a}$ & $92,75 \mathrm{a}$ & $0,88 \mathrm{~d}(0,29)$ & 6 \\
\hline Cloransulam-methyl + Glyphosate & $30,24+960$ & $27,50 \mathrm{c}$ & $62,50 \mathrm{c}$ & $82,25 \mathrm{~d}$ & $82,50 \mathrm{c}$ & $72,50 \mathrm{c}$ & $80,00 \mathrm{c}$ & $1,03 \mathrm{~d}(0,58)$ & 11 \\
\hline Chlorimuron-ethyl + Glyphosate & $12,50+960$ & $38,75 \mathrm{~b}$ & $81,25 \mathrm{~b}$ & $92,50 \mathrm{~b}$ & $96,50 \mathrm{a}$ & $93,25 \mathrm{a}$ & $95,00 \mathrm{a}$ & $0,87 \mathrm{~d}(0,27)$ & 5 \\
\hline Imazethapyr + Glyphosate & $80+960$ & $21,25 \mathrm{c}$ & $81,50 \mathrm{~b}$ & $96,50 \mathrm{a}$ & $99,25 \mathrm{a}$ & $98,75 \mathrm{a}$ & $99,50 \mathrm{a}$ & $0,72 \mathrm{~d}(0,03)$ & 1 \\
\hline Fomesafen + Glyphosate & $62,50+960$ & $55,00 \mathrm{a}$ & $85,00 \mathrm{a}$ & $97,25 \mathrm{a}$ & $98,50 \mathrm{a}$ & $97,00 \mathrm{a}$ & $97,00 \mathrm{a}$ & $0,82 \mathrm{~d}(0,18)$ & 3 \\
\hline Lactofen + Glyphosate & $72+960$ & $48,75 \mathrm{a}$ & $87,50 \mathrm{a}$ & $96,00 \mathrm{a}$ & $97,50 \mathrm{a}$ & $96,50 \mathrm{a}$ & $92,00 \mathrm{a}$ & $0,91 \mathrm{~d}(0,35)$ & 7 \\
\hline Flumiclorac-pentyl + Glyphosate & $30+960$ & $46,25 \mathrm{a}$ & $86,75 \mathrm{a}$ & $87,25 \mathrm{c}$ & $95,75 \mathrm{a}$ & $85,50 \mathrm{~b}$ & $87,75 \mathrm{~b}$ & $0,95 \mathrm{~d}(0,41)$ & 8 \\
\hline Bentazon + Glyphosate & $480+960$ & $35,00 \mathrm{~b}$ & $76,50 \mathrm{~b}$ & $93,25 \mathrm{~b}$ & $97,25 \mathrm{a}$ & $92,75 \mathrm{a}$ & $94,75 \mathrm{a}$ & $0,83 \mathrm{~d}(0,20)$ & 4 \\
\hline CV $(\%)$ & & 19,82 & 6,72 & 3,58 & 3,13 & 6,28 & 7,88 & 13,79 & \\
\hline
\end{tabular}

Valores seguidos de mesma letra nas colunas não diferem entre si pelo teste de Scott Knott p $<0,05$.

Os valores da massa seca analisados estão transformados em $\sqrt{X+0,5}$ e os valores entre parênteses são as médias originais.

Tabela 3 - Porcentagens de controle aos 3, 7, 14, 21, 28 e 35 DAA (dias após aplicação) e massa seca da parte aérea de Ipomoea grandifolia obtidas após aplicação de diferentes tratamentos contendo glyphosate no estádio de 1 a 3 folhas - Maringá-PR, 2007/2008

\begin{tabular}{|c|c|c|c|c|c|c|c|c|c|}
\hline Tratamento & Dose & \multicolumn{6}{|c|}{$\%$ Controle } & \multicolumn{2}{|c|}{ Massa seca da parte aérea } \\
\hline Ingrediente ativo & $\left(\right.$ g i.a. ou e.a. ha $\left.{ }^{-1}\right)$ & 3 DAA & $7 \mathrm{DAA}$ & 14 DAA & $21 \mathrm{DAA}$ & $28 \mathrm{DAA}$ & 35 DAA & g por vaso & $\begin{array}{c}\% \mathrm{em} \\
\text { relação à } \\
\text { testemunha }\end{array}$ \\
\hline Testemunha sem aplicação & - & $0,00 \mathrm{e}$ & $0,00 \mathrm{~g}$ & $0,00 \mathrm{~d}$ & $0,00 \mathrm{f}$ & $0,00 \mathrm{e}$ & $0,00 \mathrm{e}$ & 1,92 a $(3,23)$ & 100 \\
\hline Glyphosate & 480 & $27,50 \mathrm{c}$ & $35,50 \mathrm{e}$ & $56,25 \mathrm{~b}$ & $76,25 \mathrm{c}$ & $78,75 \mathrm{~b}$ & $78,50 \mathrm{~b}$ & $0,94 \mathrm{~d}(0,42)$ & 13 \\
\hline Cloransulam-methyl + Glyphosate & $30,24+480$ & $12,50 \mathrm{~d}$ & $43,75 \mathrm{~d}$ & $52,50 \mathrm{~b}$ & $75,00 \mathrm{c}$ & $82,50 \mathrm{~b}$ & $88,75 \mathrm{a}$ & $0,86 \mathrm{~d}(0,26)$ & 8 \\
\hline Chlorimuron-ethyl + Glyphosate & $12,50+480$ & $6,25 \mathrm{e}$ & $42,25 \mathrm{~d}$ & $46,25 \mathrm{c}$ & $67,50 \mathrm{c}$ & $78,75 \mathrm{~b}$ & $87,25 \mathrm{a}$ & $0,93 \mathrm{~d}(0,38)$ & 12 \\
\hline Imazethapyr + Glyphosate & $80+480$ & $11,25 \mathrm{~d}$ & $34,50 \mathrm{e}$ & $45,75 \mathrm{c}$ & $57,50 \mathrm{~d}$ & $73,75 \mathrm{~b}$ & $77,50 \mathrm{~b}$ & $1,03 \mathrm{~d}(0,58)$ & 18 \\
\hline Fomesafen + Glyphosate & $62,50+480$ & $47,50 \mathrm{~b}$ & $72,50 \mathrm{~b}$ & $60,75 \mathrm{~b}$ & $61,25 \mathrm{~d}$ & $57,50 \mathrm{c}$ & $61,25 \mathrm{c}$ & $1,23 \mathrm{c}(1,04)$ & 32 \\
\hline Lactofen + Glyphosate & $72+480$ & $80,75 \mathrm{a}$ & $87,50 \mathrm{a}$ & $84,50 \mathrm{a}$ & $89,75 \mathrm{a}$ & $95,25 \mathrm{a}$ & $94,75 \mathrm{a}$ & 0,75 e $(0,07)$ & 2 \\
\hline Flumiclorac-pentyl + Glyphosate & $30+480$ & $47,50 \mathrm{~b}$ & $63,25 \mathrm{c}$ & $46,25 \mathrm{c}$ & $40,00 \mathrm{e}$ & $47,50 \mathrm{~d}$ & $45,00 \mathrm{~d}$ & $1,36 \mathrm{~b}(1,35)$ & 42 \\
\hline Bentazon + Glyphosate & $480+480$ & $7,50 \mathrm{e}$ & $25,75 \mathrm{f}$ & $36,25 \mathrm{c}$ & $41,25 \mathrm{e}$ & $48,75 \mathrm{~d}$ & $40,00 \mathrm{~d}$ & $1,46 \mathrm{~b}(1,65)$ & 51 \\
\hline Glyphosate & 960 & $30,00 \mathrm{c}$ & $48,75 \mathrm{~d}$ & $69,50 \mathrm{~b}$ & $98,25 \mathrm{a}$ & $98,75 \mathrm{a}$ & $99,00 \mathrm{a}$ & 0,71 e $(0,01)$ & 0 \\
\hline Cloransulam-methyl + Glyphosate & $30,24+960$ & $6,25 \mathrm{e}$ & $27,50 \mathrm{f}$ & $58,75 \mathrm{~b}$ & $86,25 \mathrm{~b}$ & $96,50 \mathrm{a}$ & $98,75 \mathrm{a}$ & 0,73 e $(0,04)$ & 1 \\
\hline Chlorimuron-ethyl + Glyphosate & $12,50+960$ & $5,00 \mathrm{e}$ & $26,75 \mathrm{f}$ & $60,00 \mathrm{~b}$ & $90,75 \mathrm{a}$ & $97,75 \mathrm{a}$ & $98,75 \mathrm{a}$ & 0,76 e $(0,10)$ & 1 \\
\hline Imazethapyr + Glyphosate & $80+960$ & $11,25 \mathrm{~d}$ & $40,00 \mathrm{e}$ & $56,25 \mathrm{~b}$ & $85,00 \mathrm{~b}$ & $96,50 \mathrm{a}$ & $98,75 \mathrm{a}$ & 0,74 e $(0,04)$ & 2 \\
\hline Fomesafen + Glyphosate & $62,50+960$ & $53,75 \mathrm{~b}$ & $77,75 \mathrm{~b}$ & $80,00 \mathrm{a}$ & $94,50 \mathrm{a}$ & $96,50 \mathrm{a}$ & $96,75 \mathrm{a}$ & 0,76 e $(0,10)$ & 3 \\
\hline Lactofen + Glyphosate & $72+960$ & $79,50 \mathrm{a}$ & $89,75 \mathrm{a}$ & $88,25 \mathrm{a}$ & $95,75 \mathrm{a}$ & $96,75 \mathrm{a}$ & $94,75 \mathrm{a}$ & 0,76 e $(0,10)$ & 3 \\
\hline Flumiclorac-pentyl + Glyphosate & $30+960$ & $48,75 \mathrm{~b}$ & $72,50 \mathrm{~b}$ & $61,25 \mathrm{~b}$ & $80,50 \mathrm{~b}$ & $82,75 \mathrm{~b}$ & $80,75 \mathrm{~b}$ & $0,93 \mathrm{~d}(0,37)$ & 11 \\
\hline Bentazon + Glyphosate & $480+960$ & $6,25 \mathrm{e}$ & $18,25 \mathrm{f}$ & $40,00 \mathrm{c}$ & $72,50 \mathrm{c}$ & $80,75 \mathrm{~b}$ & $86,00 \mathrm{a}$ & $0,95 \mathrm{~d}(0,44)$ & 14 \\
\hline CV $(\%)$ & & 16,66 & 12,05 & 12,14 & 7,87 & 5,77 & 11,40 & 10,90 & \\
\hline
\end{tabular}

Valores seguidos de mesma letra nas colunas não diferem entre si pelo teste de Scott Knott $\mathbf{p}<0,05$.

Os valores da massa seca analisados estão transformados em $\sqrt{X+0,5}$ e os valores entre parênteses são as médias originais. 
dos niveis de controle (35 DAA) de I. grandifolia. $\mathrm{Na}$ mistura com imazethapyr, os resultados não diferiram significativamente em relação ao glyphosate isolado, mostrando não haver incremento ou decréscimo no controle quando combinado com a menor dose de glyphosate.

Na dose de 960 g e.a. ha-1, o glyphosate somente apresentou interação negativa quando em mistura com flumiclorac-pentyl (80,75\%). Para as demais misturas, não houve diferenças significativas em relação à aplicação isolada.

Assim, fica clara a ocorrência da interação positiva entre o glyphosate a 480 g e.a. ha ${ }^{1} \mathrm{com}$ cloransulam-methyl, lactofen e chlorimuronethyl para o controle de I. grandifolia no estádio de 1 a 3 folhas, semelhante a resultados obtidos por Procópio et al. (2007), que mostraram que o glyphosate na dose de $1.440 \mathrm{~g}$ e.a. ha ${ }^{-1}$ com 5,0 ou $10,0 \mathrm{~g} \mathrm{ha}^{-1}$ de chlorimuron-ethyl foi mais eficiente no controle de I. grandifolia com 2 a 6 folhas, se comparado ao glyphosate isolado.

Comparando os resultados das misturas de glyphosate com os dos demais herbicidas, nota-se que na dose de $480 \mathrm{~g}$ e.a. ha ${ }^{-1}$ o controle sofreu maior influência dos herbicidas em mistura, se comparado à dose de 960 g e.a. ha-1

Os tratamentos com glyphosate a 480 e 960 g e.a. ha ${ }^{-1}$ isolado ou em mistura com os outros herbicidas apresentaram massa seca da parte aérea máxima de 51 e 14\%, respectivamente, em relação à testemunha sem aplicação, mostrando que os tratamentos com maior dose de glyphosate, de modo geral, foram mais eficientes. Norsworthy et al. (2001) encontraram resultados semelhantes para Ipomoea lacunosa ao verificarem $71 \%$ de redução da massa seca em relação à testemunha sem aplicação quando as plantas foram tratadas com glyphosate a 1.120 g e.a. ha ${ }^{-1}$.

\section{Ipomoea grandifolia no estádio de 4 a 6 folhas}

Para I. grandifolia no estádio de 4-6 folhas o glyphosate em aplicação isolada apresentou eficiência crescente no controle a partir de 3 DAA nas duas doses (480 e 960 g e.a. ha-1), com controles finais de 63,75 e 83,75\%, respectivamente (Tabela 4); no entanto, mesmo na maior dose utilizada o controle não foi considerado ótimo, demonstrando uma tolerância dessa espécie quando o herbicida é aplicado em estádios de desenvolvimento mais avançados. Para Monquero et al. (2004), a absorção do glyphosate envolve uma rápida penetração inicial através da cutícula, seguida por uma absorção simplástica lenta; a duração desse processo é dependente de vários fatores, como espécie e idade da planta, condições ambientais e concentração do herbicida na calda. Esses autores afirmam que para I. grandifolia a tolerância ao herbicida ${ }^{14} \mathrm{C}$ glyphosate ocorre devido à menor translocação, uma vez que, 72 horas após o tratamento, havia penetrado $80 \%$ do produto aplicado; contudo, do ${ }^{14} \mathrm{C}$ glyphosate absorvido, 68,5\% encontrava-se na folha que recebeu a aplicação e somente 2,2\% nas demais folhas, $3,5 \%$ no caule e $4,6 \%$ nas raízes.

O uso das misturas de glyphosate a 480 g e.a. ha ${ }^{-1}$ com os produtos cloransulammethyl, chlorimuron-ethyl, flumiclorac-pentyl, bentazon e imazethapyr não proporcionou nenhum ganho significativo em termos de controle final quando comparado ao glyphosate isolado. A mistura dessa mesma dose com fomesafen apresentou menor controle em relação ao produto isolado. A mistura de lactofen com glyphosate (480 g e.a. ha ${ }^{-1}$ ) foi significativamente superior ao controle do glyphosate isolado e também às demais misturas com essa mesma dose.

Nas avaliações das misturas de glyphosate a 960 g e.a. ha ${ }^{-1}$ com cloransulam-methyl, fomesafen, lactofen, chlorimuron-ethyl, flumiclorac-pentyl, bentazon e imazethapyr, verificaram-se valores crescentes nos controles a partir de 3 DAA, chegando aos 35 DAA com 96,25\%, 98,00\%, 98,25\%, 96,50\%, $96,00 \%, 96,00 \%$ e $99,50 \%$, respectivamente, sendo todos eles superiores ao glyphosate isolado. Os tratamentos contendo glyphosate (960 g e.a. ha-1) em mistura com os demais produtos foram superiores às duas doses de glyphosate isoladamente, semelhante ao constatado por Ferreira Neto et al. (2009), os quais obtiveram controle de $99,2 \%$ para plantas de I. grandifolia, aos 30 DAA da mistura de glyphosate (960 g e.a. ha ${ }^{-1}$ ) cloransulammethyl (40 $\left.\mathrm{g} \mathrm{ha}^{-1}\right)$, no estádio de desenvolvimento de 2 a 6 folhas. 
Tabela 4 - Porcentagens de controle aos 3, 7, 14, 21, 28 e 35 DAA (dias após aplicação) e massa seca da parte aérea de Ipomoea grandifolia obtidas após aplicação de diferentes tratamentos contendo glyphosate no estádio de 4 a 6 folhas - Maringá-PR, 2007/2008

\begin{tabular}{|c|c|c|c|c|c|c|c|c|c|}
\hline Tratamento & Dose & \multicolumn{6}{|c|}{$\%$ Controle } & \multicolumn{2}{|c|}{ Massa seca da parte aérea } \\
\hline Ingrediente ativo & (g i.a. ou e.a. ha $\left.{ }^{-1}\right)$ & 3 DAA & 7 DAA & 14 DAA & 21 DAA & 28 DAA & 35 DAA & g por vaso & $\begin{array}{c}\% \text { em } \\
\text { relação à } \\
\text { testemunha }\end{array}$ \\
\hline Testemunha sem aplicação & - & $0,00 \mathrm{e}$ & $0,00 \mathrm{~h}$ & $0,00 \mathrm{i}$ & $0,00 \mathrm{~g}$ & $0,00 \mathrm{e}$ & $0,00 \mathrm{e}$ & 2,42 a $(5,40)$ & 100 \\
\hline Glyphosate & 480 & $5,00 \mathrm{e}$ & $16,25 \mathrm{~g}$ & $32,50 \mathrm{~h}$ & $18,75 \mathrm{f}$ & $33,75 \mathrm{~d}$ & $63,75 \mathrm{c}$ & $1,79 \mathrm{~b}(2,73)$ & 51 \\
\hline Cloransulam-methyl + Glyphosate & $30,24+480$ & $10,00 \mathrm{~d}$ & $35,00 \mathrm{e}$ & $41,25 \mathrm{~g}$ & $46,25 \mathrm{e}$ & $41,25 \mathrm{~d}$ & $58,75 \mathrm{c}$ & $1,51 \mathrm{~b}(1,79)$ & 33 \\
\hline Chlorimuron-ethyl + Glyphosate & $12,50+480$ & $5,00 \mathrm{e}$ & $33,75 \mathrm{e}$ & $53,75 \mathrm{e}$ & $64,50 \mathrm{~d}$ & $51,25 \mathrm{c}$ & $66,25 \mathrm{c}$ & $1,62 \mathrm{~b}(2,16)$ & 40 \\
\hline Imazethapyr + Glyphosate & $80+480$ & $5,00 \mathrm{e}$ & $25,00 \mathrm{f}$ & $55,75 \mathrm{e}$ & $62,00 \mathrm{~d}$ & $48,75 \mathrm{c}$ & $58,75 \mathrm{c}$ & $1,59 \mathrm{~b}(2,08)$ & 39 \\
\hline Fomesafen + Glyphosate & $62,50+480$ & $30,00 \mathrm{c}$ & $49,00 \mathrm{~d}$ & $77,00 \mathrm{c}$ & $73,25 \mathrm{c}$ & $46,25 \mathrm{c}$ & $46,25 \mathrm{~d}$ & $1,72 \mathrm{~b}(2,50)$ & 46 \\
\hline Lactofen + Glyphosate & $72+480$ & $27,50 \mathrm{c}$ & $68,75 \mathrm{c}$ & $86,25 \mathrm{~b}$ & $89,25 \mathrm{~b}$ & $85,00 \mathrm{~b}$ & $84,00 \mathrm{~b}$ & $1,23 \mathrm{c}(1,13)$ & 21 \\
\hline Flumiclorac-pentyl + Glyphosate & $30+480$ & $30,00 \mathrm{c}$ & $46,25 \mathrm{~d}$ & $73,00 \mathrm{~d}$ & $77,00 \mathrm{c}$ & $55,00 \mathrm{c}$ & $58,25 \mathrm{c}$ & $1,62 \mathrm{~b}(2,15)$ & 40 \\
\hline Bentazon + Glyphosate & $480+480$ & $7,50 \mathrm{~d}$ & $26,25 \mathrm{f}$ & $60,50 \mathrm{e}$ & $68,25 \mathrm{~d}$ & $46,25 \mathrm{c}$ & $57,50 \mathrm{c}$ & $1,58 \mathrm{~b}(2,01)$ & 37 \\
\hline Glyphosate & 960 & $10,00 \mathrm{~d}$ & $26,25 \mathrm{f}$ & $47,50 \mathrm{f}$ & $65,00 \mathrm{~d}$ & $76,25 \mathrm{~b}$ & $83,75 \mathrm{~b}$ & $1,32 \mathrm{c}(1,28)$ & 24 \\
\hline Cloransulam-methyl + Glyphosate & $30,24+960$ & $11,25 \mathrm{~d}$ & $33,75 \mathrm{e}$ & $77,00 \mathrm{c}$ & $88,25 \mathrm{~b}$ & $83,75 \mathrm{~b}$ & $96,25 \mathrm{a}$ & $0,96 \mathrm{~d}(0,43)$ & 8 \\
\hline Chlorimuron-ethyl + Glyphosate & $12,50+960$ & $10,00 \mathrm{~d}$ & $47,00 \mathrm{~d}$ & $77,00 \mathrm{c}$ & $88,00 \mathrm{~b}$ & $88,00 \mathrm{~b}$ & $96,50 \mathrm{a}$ & $0,99 \mathrm{~d}(0,53)$ & 10 \\
\hline Imazethapyr + Glyphosate & $80+960$ & $5,00 \mathrm{e}$ & $38,75 \mathrm{e}$ & $90,00 \mathrm{~b}$ & $98,00 \mathrm{a}$ & $99,25 \mathrm{a}$ & $99,50 \mathrm{a}$ & $0,71 \mathrm{~d}(0,00)$ & 0 \\
\hline Fomesafen + Glyphosate & $62,50+960$ & $52,50 \mathrm{a}$ & $85,00 \mathrm{a}$ & $95,50 \mathrm{a}$ & $97,25 \mathrm{a}$ & $94,00 \mathrm{a}$ & $98,00 \mathrm{a}$ & $0,90 \mathrm{~d}(0,32)$ & 6 \\
\hline Lactofen + Glyphosate & $72+960$ & $45,00 \mathrm{~b}$ & $77,50 \mathrm{~b}$ & $96,25 \mathrm{a}$ & $97,50 \mathrm{a}$ & $96,50 \mathrm{a}$ & $98,25 \mathrm{a}$ & $0,83 \mathrm{~d}(0,19)$ & 4 \\
\hline Flumiclorac-pentyl + Glyphosate & $30+960$ & $31,25 \mathrm{c}$ & $75,00 \mathrm{~b}$ & $81,25 \mathrm{c}$ & $92,75 \mathrm{a}$ & $92,50 \mathrm{a}$ & $96,00 \mathrm{a}$ & $1,09 \mathrm{c}(0,69)$ & 13 \\
\hline Bentazon + Glyphosate & $480+960$ & $8,75 \mathrm{~d}$ & $45,00 \mathrm{~d}$ & $68,75 \mathrm{~d}$ & $82,50 \mathrm{~b}$ & $84,25 \mathrm{~b}$ & $96,00 \mathrm{a}$ & $1,16 \mathrm{c}(0,86)$ & 16 \\
\hline $\mathrm{CV}(\%)$ & & 21,91 & 10,62 & 8,36 & 8,05 & 11,73 & 10,01 & 14,07 & \\
\hline
\end{tabular}

Valores seguidos de mesma letra nas colunas não diferem entre si pelo teste de Scott Knott p $<0,05$.

Os valores da massa seca analisados estão transformados em $\sqrt{X+0,5} \mathrm{e}$ os valores entre parênteses são as médias originais.

Analisando os valores de massa seca da parte aérea, nota-se maior eficiência do glyphosate (960 g e.a. ha ${ }^{-1}$ ) isolado e em mistura com os demais herbicidas, com valores entre 0 e $16 \%$ em relação à testemunha sem aplicação (Tabela 4).

Esses resultados corroboram os de Norsworthy \& Grey (2004), que, ao avaliarem a adição de chlorimuron-ethyl ( $6 \mathrm{e} 9 \mathrm{~g} \mathrm{ha}^{-1}$ ) ao glyphosate (420 e 840 g e.a. ha ${ }^{-1}$ ), observaram eficiência de controle superior (85 e 97\%, respectivamente) quando comparado com a aplicação de glyphosate isoladamente (83 e $84 \%$, respectivamente).

Em resumo, para ambas as espécies avaliadas, a utilização da dose de 960 g e.a. ha ${ }^{-1}$ do glyphosate dispensa a necessidade de misturas com outros herbicidas, quando os tratamentos são aplicados no estádio de 1-3 folhas (Tabela 5). No entanto, ao se utilizar a dose de 480 g e.a. ha- ${ }^{-1}$ do glyphosate, a adição de outros herbicidas pode afetar negativamente o controle de E. heterophylla para o controle de I. grandifolia, os herbicidas em mistura apresentaram comportamento distinto, sendo semelhante, superior ou inferior ao do glyphosate a 480 g e.a. ha ${ }^{-1}$ isolado. Para aplicações realizadas em E. heterophylla no estádio de 4-6 folhas, aumentos da eficácia de controle em relação ao glyphosate aplicado isoladamente a $480 \mathrm{~g}$ e.a. ha ${ }^{-1}$ são observados com as misturas com inibidores de protox (fomesafen, lactofen e flumiclorac-pentyl). Para I. grandifolia em estádio de 4-6 folhas, os herbicidas fomesafen e flumiclorac-pentyl reduziram a eficácia de controle quando adicionados ao glyphosate a 480 g e.a. ha ${ }^{-1}$. Já para o glyphosate a 960 g e.a. ha ${ }^{-1}$, todos os herbicidas em mistura proporcionaram incrementos na eficácia de controle (Tabela 5).

De modo geral, os controles das espécies E. heterophylla e I. grandifolia foram melhores quando os tratamentos foram aplicados no estádio de 1-3 folhas tanto pelo herbicida glyphosate isolado (480 e 960 g e.a. ha-1) como em mistura com os demais herbicidas. No estudo realizado, verificou-se que o comportamento das misturas de glyphosate com outros herbicidas pode variar conforme a dose de glyphosate, o herbicida utilizado em mistura, 
Tabela 5 - Resumo dos resultados de controle (35 DAA) obtidos com as misturas de glyphosate com outros herbicidas em relação a Euphorbia heterophylla e Ipomoea grandifolia em dois estádios de desenvolvimento - Maringá-PR, 2007/2008

\begin{tabular}{|c|c|c|c|c|c|c|c|c|}
\hline \multirow{4}{*}{ Herbicidas } & \multicolumn{4}{|c|}{ Euphorbia heterophylla } & \multicolumn{4}{|c|}{ Ipomoea grandifolia } \\
\hline & \multicolumn{2}{|c|}{ 1-3 folhas } & \multicolumn{2}{|c|}{ 4-6 folhas } & \multicolumn{2}{|c|}{ 1-3 folhas } & \multicolumn{2}{|c|}{ 4-6 folhas } \\
\hline & \multicolumn{8}{|c|}{ Doses do glyphosate (g e.a. ha $^{-1}$ ) } \\
\hline & 480 & 960 & 480 & 960 & 480 & 960 & 480 & 960 \\
\hline Cloransulam-methyl & $=$ & $=$ & $=$ & - & + & $=$ & $=$ & + \\
\hline Chlorimuron-ethyl & $=$ & $=$ & $=$ & $=$ & + & $=$ & $=$ & + \\
\hline Imazethapyr & - & $=$ & $=$ & $=$ & $=$ & $=$ & $=$ & + \\
\hline Fomesafen & - & $=$ & + & $=$ & - & $=$ & - & + \\
\hline Lactofen & - & $=$ & + & $=$ & + & $=$ & ++ & + \\
\hline Flumiclorac-pentyl & - & $=$ & + & - & -- & - & $=$ & + \\
\hline Bentazon & - & $=$ & -- & $=$ & -- & $=$ & $=$ & + \\
\hline
\end{tabular}

= interação não significativa; - interação negativa e + interação positiva em relação à aplicação isolada de glyphosate nas doses de 480 e 960 g e.a. ha ${ }^{-1}$; - -: redução de controle $\geq 20 \%$ em relação às doses isoladas de glyphosate; ++: acréscimos de controle $\geq 20 \%$ em relação às doses isoladas de glyphosate.

a espécie e o estádio de desenvolvimento da planta daninha.

\section{LITERATURA CITADA}

ALONSO, D. G. Seletividade de glyphosate isolado ou em mistura para soja RR. 2008. 72 f. Dissertação (Mestrado em Proteção dePlantas) - Universidade Estadual de Maringá, Maringá, 2008

FERREIRA, E. A. et al. Estudos anatômicos de folhas de espécies de plantas daninhas de grande ocorrência no Brasil. IV - Amaranthus deflexus, Amaranthus spinosus, Alternanthera tenella e Euphorbia heterophylla.

Planta Daninha, v. 21, n. 2, p. 263-271, 2003.

GAZZIERO, D. L. P. et al. Deposição de glyphosate aplicado para controle de plantas daninhas em soja transgênica.

Planta Daninha, v. 24, n. 1, p. 173-181, 2006.

GONZINI, L. C.; HART, E. S.; WAX, L. M. Herbicide combination for weed management in glyphosate-resistant soybean. Weed Technol., v. 13, n. 2, p. 354-360, 1999.

KRANZ, R. F. et al. Influence of weeds competition and herbicides on glyphosate resistAnt soybean (Glycine max). Weed Technol., v. 15, n. 3, p. 530-534, 2001.

MONQUERO, P. A.; CHRISTOFFOLETI, P. J.; SANTOS, C. T. D. Glyphosate em mistura com herbicidas alternativos para o manejo de plantas daninhas. Planta Daninha, v. 19, n. 3, p. 375-380, 2001.

MONQUERO, P. A. et al. Absorção, translocação e metabolismo do glyphosate por plantas tolerantes e susceptíveis a este herbicida. Planta Daninha, v. 22, n. 3, p. 445-451, 2004.
FERREIRA NETO, M. E. et al. Seletividade de herbicidas pós-emergentes aplicados na soja geneticamente modificada. Planta Daninha, v. 27, n. 2, p. 345-352, 2009.

NORSWORTHY, J. K., BURGOS, N. R.; OLIVER, L. R. Differences in weed tolerance to glyphosate involve different mechanisms. Weed Technol., v. 15, n. 3, p. 725-731, 2001.

NORSWORTHY, J. K.; GREY, T. L. Addition of nonionic surfactant to glyphosate plus chlorimuron. Weed Technol., v. 18, n. 3 , p. $588-593,2004$

PROCÓPIO, S. O. et al. Utilização de chlorimuron-ethyl e imazethapyr na cultura da soja Roundup Ready ${ }^{\circledR}$.

Planta Daninha, v. 25, n. 2, p. 365-373, 2007.

PETTER, F. A. et al. Manejo de herbicidas na cultura da soja Roundup Ready ${ }^{\circledR}$. Planta Daninha, v. 25, n. 3, p. 557-566, 2007.

SHAW, D. R.; ARNOLD, J. C. Weed control from herbicide combinations with glyphosate. Weed Technol., v. 16, n. 1, p. $1-6,2002$.

SCOTT, A.; KNOTT, M. Cluster-analysis method for grouping means in analysis of variance. Biometrics, v. 30, n. 3, p. 507-512, 1974.

SOCIEDADE BRASILEIRA DA CIÊNCIA DAS PLANTAS DANINHAS - SBCPD. Procedimentos para instalação, avaliação e análise de experimentos com herbicidas. Londrina: $1995.42 \mathrm{p}$.

VIDAL, R. A. et al. Antagonismo na associação de glyphosate e triazinas. Planta Daninha, v. 21, n. 2, p. 301-306, 2003.

VIDRINE, R. P.; GRIFFIN, J. L.; BLOUIN, D. C. Evaluation of reduced rates of glyphosate and chlorimuron in glyphosateresistant soybean (Glycine max). Weed Technol., v. 16, n. 4, p. 731-736, 2002. 